Aus aktuellem Anlass

Gabriel J. Felbermayr*

\title{
Zur Rückkehr der Machtpolitik in Handelsfragen: Theoretische Überlegungen und politische Empfehlungen
}

https://doi.org/10.1515/pwp-2018-0027

Zusammenfassung: Opportunistische Regierungen haben vielfache Anreize, unilateral vom Freihandel abzuweichen, zum Beispiel um ihre Terms of trade zu verbessern. Doch was individuell rational sein mag, führt kollektiv in eine Situation, in der alle Länder schlechter gestellt sind, und aus der sie nur schwer herausfinden. Die neuere Forschung zeigt, dass es das Prinzip der Reziprozität im GATT/WTO-System ermöglicht hat, aus diesem Gefangenendilemma auszubrechen. Das Prinzip stellt sicher, dass bei Zollsenkungen die Terms of trade der Länder konstant bleiben, so dass alle Beteiligten profitieren. Reziprozität bedeutet auch, dass der Bruch von Vereinbarungen durch streng reglementierte „Gegenzölle“ kompensiert werden darf. Die glaubwürdige Drohung mit solchen Maßnahmen ist das einzige Mittel, opportunistisches Verhalten in Schach zu halten und die Stabilität der multilateralen Ordnung zu bewahren. Daher ist es auch richtig, dass die EU auf die unter dem Vorwand der Bedrohung der nationalen Sicherheit erhobenen amerikanischen Zölle auf Stahl und Aluminium mit maßvollen Vergeltungszöllen reagiert hat.

JEL-Klassifikation: F13, F55, C71

Schlüsselwörter: Handelspolitik, Optimalzolltheorie, Spieltheorie

\section{Theoretische Vorbemerkungen}

\subsection{Der wohlfahrtsökonomisch optimale Zollsatz liegt typischerweise nicht bei Null}

Zölle sind Steuern auf importierte Güter. Sie dienen dazu, ausländische Anbieter gegenüber inländischen zu diskri-

*Kontaktperson: Gabriel J. Felbermayr, Ludwig Maximilians Universität München und ifo Institut, Poschingerstraße 5, 81679 München, E-Mail: felbermayr@ifo.de minieren, und damit importierte Güter gegenüber importierten Dienstleistungen, die gewöhnlich nicht verzollt werden (es sei denn indirekt, als Bestandteil importierter Güter). Zölle verzerren die Konsumentscheidungen der Haushalte - anders als eine allgemeine Verbrauchsteuer und die Beschaffungsentscheidungen von Unternehmen. Zölle sind also vergleichsweise „schlechte“ Steuern. Es gibt deutlich effizientere, sprich: verzerrungsärmere Wege, die Finanzierung öffentlicher Aufgaben sicherzustellen. Es gibt daher einen relativ ausgeprägten Konsens unter Ökonomen, Zölle wegen der mit ihnen verbundenen Wohlfahrtskosten abzulehnen.

In einer Welt, in der alle Länder auf Zölle verzichten, erreichen alle Länder typischerweise höhere Wohlfahrtsniveaus. ${ }^{1}$ Das heißt nun aber nicht, dass es aus der Sicht eines einzelnen Landes immer optimal ist, auf Zölle gänzlich zu verzichten. ${ }^{2}$ Das gilt nicht einmal dann, wenn man annimmt, dass eine Regierung keine anderen Ziele verfolgt als die Maximierung der gesamtwirtschaftlichen Wohlfahrt ihres Landes. Nur in einem Land, das so klein ist, dass seine Zollpolitik keinerlei Einfluss auf die Verkaufspreise der ausländischen Anbieter hat, ist der wohlfahrtsmaximierende Zollsatz Null. Für große Länder gilt dies nicht.

Ein Importzoll senkt im Normalfall die Nachfrage nach ausländischen Gütern. Wenn die ausländischen Exporteure, bildlich gesprochen, auf einer steigend verlaufenden Angebotsfunktion unterwegs sind, dann senkt der Zoll ihren Verkaufspreis; das importierende Land muss weni-

1 In simplen Modellen mit nur zwei (relativ symmetrischen) Ländern und einfach strukturierten Güterräumen gilt, dass alle Länder ohne Zölle besser gestellt sind als mit Zöllen. Man kann allerdings durchaus Modelle konstruieren, in denen zwar die Welt insgesamt ohne Zölle eine höhere Wohlfahrt erreicht, einzelne Länder aber durch Abschaffung von Zöllen verlieren; siehe beispielsweise Kennan und Riezman 1988. Die Bedingungen für solche Ergebnisse sind allerdings sehr unrealistisch.

2 Das in der Folge diskutierte Optimalzollargument geht auf Mill (1844) und Bickerdike (1907) zurück. Johnson (1953) bietet die erste moderne Behandlung des Themas. 
ger für die Waren bezahlen, und der Preis für die Konsumenten steigt um weniger als um den Zoll. ${ }^{3}$ Diese Verbesserung der internationalen Austauschverhältnisse (Terms of trade) erhöht die gesamtwirtschaftliche Wohlfahrt. Daneben erzeugt der Zoll auch erhebliche Umverteilungseffekte innerhalb des zollerhebenden Landes: Die Verbraucher (ob Konsumenten oder Unternehmen) werden belastet, der Staat generiert Einnahmen, und die heimischen Produzenten des verzollten Gutes profitieren von höheren Preisen. Für ein großes Land ist der „Optimalzoll“ also nicht Null. Er kann aber auch nicht beliebig hoch sein, denn dann überwiegen die verzerrenden Effekte des Zolls den positiven Terms-of-trade-Effekt.

Das Optimalzollargument lässt sich übrigens auch im Partialmarktmodell schön demonstrieren. Wie bei jeder Steuer fallen auch bei Zöllen die Zahllast und die Traglast nicht zusammen. Typischerweise entrichtet der Importeur eines Gutes den Zoll an die zuständige heimische Behörde, und dieser Zoll treibt einen Keil zwischen den Preis, den die ausländischen Erzeuger erhalten, und den Preis, den die inländischen Erwerber bezahlen. Die Zahllast entfällt also auf die Importeure. Doch wie bei jeder anderen Steuer ist davon auszugehen, dass ein Teil des Zolles auf die ausländischen Erzeuger überwälzt wird: Hersteller und Erwerber teilen sich die Traglast.

In welchem Ausmaß diese Überwälzung stattfindet, hängt von den Elastizitäten ab. Wenn die Preiselastizität des ausländischen Angebots größer ist als die Preiselastizität der inländischen Nachfrage, dann zahlen die heimischen Konsumenten den größeren Anteil. Wenn das ausländische Angebot weniger preiselastisch ist als die inländische Nachfrage, dann entfällt die Zahllast hauptsächlich auf die ausländischen Anbieter. Im Extremfall, wenn die heimische Nachfrage überhaupt nicht auf Preissteigerungen reagiert, müssen die heimischen Konsumenten den Zoll zur Gänze bezahlen. Wenn aber das ausländische Angebot überhaupt nicht vom Preis abhängt, dann bezahlen die Anbieter den Zoll zur Gänze. Bei den allermeisten Gütern ist von einer gewissen Überwälzung auszugehen. Das heißt, Importzölle erlauben den Griff in die Tasche ausländischer Steuersubjekte. Sie stellen „Beggarthy-neighbour“-Politik dar, wie schon Adam Smith (1776) erkannte: Das exportierende Land verarmt, weil der relative Preis seines Exportgutes sinkt; das importierende Land profitiert, weil es billiger an die Importe kommt.

3 Wenn das so genannte Metzler-Paradox (Metzler 1949) vorliegt, fällt der Produzentenpreis so stark, dass auch der Konsumentenpreis inklusive Zoll niedriger ist.
In der neueren Außenwirtschaftstheorie - zum Beispiel im Modell von Krugman (1980) mit Produktdifferenzierung, Größenvorteilen und monopolistischem Wettbewerb - gibt es auch für ein „kleines“ Land einen wohldefinierten Optimalzoll, weil hier jeder Produzent in seiner engen Marktnische über Marktmacht verfügt. Dies hat Gros (1987) gezeigt. Das lässt sich am leichtesten verstehen, wenn man die Lerner-Symmetrie zu Hilfe zieht. ${ }^{4}$ Diese besagt, dass bei Gültigkeit einer Budgetrestriktion die Wirkung eines Importzolls immer gleich der Wirkung einer Exportsteuer ist. Wenn also ein kleines Land seine Importe mit Zöllen verteuert und dadurch reduziert, müssen auch seine Exporte zurückgehen, und dies bedeutet im internationalen Gleichgewicht, dass sich seine Terms of trade verbessert haben müssen. ${ }^{5}$

Wenn man das Krugman-Modell um heterogene Firmen und Selektionseffekte (nicht jedes Unternehmen exportiert) erweitert (Melitz 2003), verkompliziert sich die Situation etwas. Zölle beeinflussen nun auch, ob Unternehmen überhaupt exportieren. Gleichwohl gilt das Resultat von Gros (1987) weiterhin, wie Felbermayr et al. (2013) gezeigt haben. Wenn es Transportkosten gibt, erhöht der Selektionseffekt den Optimalzoll sogar, weil die Anzahl der im Inland hergestellten Varianten steigt und jene der importierten Varianten fällt. Somit werden die heimischen Verbraucher von Transportkosten entlastet. Dieses Standortargument existiert auch in Modellen der neuen ökonomischen Geographie, zum Beispiel von Venables (1987), und jüngst hat es auch Ossa (2011) in einem eleganten Modell gezeigt. In Modellen mit variablen Gewinnspannen, zum Beispiel in Cournot-Oligopolen, gibt es auch ein Profit-shifting-Argument: Durch Zölle können Gewinne ins Inland verlagert werden. Bagwell und Staiger (2012) zeigen jedoch, dass auch diese „alternativen“ Optimalzollargumente letztlich als Manipulation der Terms of trade verstanden werden können. Die Crux: Auch „kleine“ Länder können Marktmacht haben, und dies bedeutet, dass ihre unilateral optimalen Zölle größer als Null sind. Außerdem gilt in allen - neueren und älteren - Modellen, dass größere Länder höhere Zölle setzen.

In der Literatur werden nicht-tarifäre Handelskosten häufig als so genannte Eisberge modelliert. Beim Transport von Gütern schmelzen diese. Es muss eine größere Menge im Quellland verschickt werden, damit eine bestimmte Menge eines Gutes im Zielland ankommt. Solche Handelskosten sind gesamtwirtschaftlich immer schäd-

4 Lerner 1936; eine moderne Darstellung findet sich in Costinot und Werning 2018.

5 Für das Argument ist es nicht notwendig, dass die Länder ausgeglichene Handelsbilanzen aufweisen. 
lich; es ergibt nie Sinn, sie künstlich zu erhöhen. Solche Handelskosten, die kein Zolleinkommen generieren, sind auch aus unilateraler Sicht optimalerweise immer Null. ${ }^{6}$ Bei der Wahl von Regulierung auf globalen Märkten kann es allerdings durchaus zu opportunistischen Beggar-thyneighbour-Situationen kommen, zum Beispiel wenn eine Regierung versucht, ein umweltpolitisches Ziel durch besonders harte Regulierung ausländischer Güter bei gleichzeitiger Schonung inländischer Hersteller zu erreichen. Auch hier gibt es die Versuchung, die Kosten der Regulierung Ausländern aufzubürden, während die Vorteile den Inländern zukommen.

\subsection{Was motiviert Politiker wirklich?}

Die Optimalzolltheorie wird gerne als unrealistisch abgetan, und zwar gleich aus mehreren Gründen. Erstens begründen Politiker ihre Handelspolitik offiziell nicht mit Terms-of-trade-Effekten. Das Standortargument der neueren Außenhandelstheorie hingegen taucht schon eher auf, zum Beispiel in den Tweets des amerikanischen Präsidenten Donald Trump. Zweitens fragt sich: Wenn Politiker die internationalen Austauschverhältnisse manipulieren wollen, warum verwenden sie dann keine Exportsteuern, die ja, der Lerner-Symmetrie zufolge, die exakt gleichen Effekte wie Importzölle haben sollten? Und warum sind Abmachungen so beliebt, bei denen ein importierendes Land seine Handelspartner nötigt, „freiwillig“ die Exportmengen zu beschränken, obwohl diese die Terms of trade verschlechtern? ${ }^{7}$

Die Theorie kollektiver Entscheidungen von Olson (1965) ist wahrscheinlich besser geeignet, die tatsächliche Zollpolitik zu erklären: Importzölle schaden Millionen von Konsumenten in sehr geringem Ausmaß, nutzen aber einigen wenigen Produzenten enorm. Letztere haben große Anreize, in die Beeinflussung von Politik zu investieren, und ihre geringe Zahl reduziert das Problem des Trittbrettfahrens. Die Konsumenten hingegen haben nur geringe Anreize, gegen Zölle aufzutreten, weil der Schaden für den Einzelnen zu gering ist. Grossman und Helpman (1994)

6 Nur in Modellen, in denen die Faktorpreise fixiert und die Firmen international mobil sind, können unilateral höhere Eisbergkosten die Wohlfahrt eines Landes erhöhen. Im allgemeinen Gleichgewicht ist dies aber nicht möglich (siehe Felbermayr und Jung 2012).

7 Solche freiwilligen Exportbeschränkungen galten in den achtziger Jahren zwischen den Vereinigten Staaten und Japan in der Automobilindustrie. Gegenwärtig haben sich Argentinien, Brasilien und Korea zu Beschränkungen ihrer Stahlexporte in die Vereinigten Staaten verpflichtet. gehen einen Schritt weiter und argumentieren, dass Protektionismus politisch „erkauft“ werden kann. Damit formalisieren sie Olsons Theorie. Die nachfolgende Literatur zeigt, dass Lobbyaktivitäten in der Tat die beobachtete Handelspolitik sehr gut erklären. Gleichzeitig gibt es aber auch überzeugende empirische Evidenz dafür, dass ein Land seine Importzölle dort hoch wählt, wo es in der Tat Marktmacht hat (Broda et al. 2008). Und selbst wenn die Politiker nicht die allgemeine Wohlfahrt zu maximieren suchen, sondern ihre Wiederwahlwahrscheinlichkeit oder einfach nur ihr Einkommen, dann setzen sie dort höhere Zölle, wo ihr Land über mehr Marktmacht verfügt.

Ganz abgesehen davon ist es für die ökonomische Wirkungsanalyse nicht entscheidend, wie die Regierungen verschiedener Länder ihre Zollpolitik motivieren. Zentral ist vielmehr, dass sie unilateral Anreize haben, Importzölle einzusetzen, und dass sie damit ihren Handelspartnern schaden.

\subsection{Individuell rational, kollektiv schädlich}

Kehren wir zum Optimalzollargument zurück. Für die Regierungen aller Länder ist es individuell rational, ihre Terms of trade mit Hilfe von Importzöllen (oder Exportsteuern) zu ihrem Vorteil zu manipulieren. Der Importzoll des einen Landes reduziert nun den Preis des Importgutes relativ zum Preis des eigenen Exportguts. Der Importzoll des anderen Landes tut genau das gleiche, nur dass hier die Rollen des Export- und des Importgutes vertauscht sind. Die Folge ist, dass die Austauschverhältnisse beider Länder unverändert bleiben: Der jeweilige Handelspartner verhindert mit seiner opportunistischen Politik die Termsof-trade-Verbesserung des anderen. Was bleibt, sind die verzerrenden Effekte der Zölle. Wenn alle Länder Importzölle erheben, stellen sie sich alle schlechter, als wenn sie gemeinsam auf Zölle verzichteten. Besser wäre es, alle Länder verzichten auf Zölle.

Das Problem liegt hier in einem klassischen Gefangenendilemma. Wenn ein Land auf seine Zölle verzichtete, müsste es eine Verschlechterung seiner Terms of trade in Kauf nehmen. Angesichts der Zölle der Handelspartner ist es also individuell nicht rational, den Außenhandel zu liberalisieren. Die Abweichung von der kooperativen Strategie (Nullzölle) ist die dominante Strategie. Was es braucht, ist ein geeignetes System, das das gleichzeitige Senken aller Zölle ermöglicht und dabei die Terms of trade konstant hält.

Im Gefangenendilemma setzen alle Spieler ihre Zölle so, dass sie in optimaler Weise auf die Zölle aller anderen Spieler reagieren. Die resultierenden Zölle in einem sol- 
chen Nash-Gleichgewicht sind erheblich. In der Arbeit von Felbermayr et al. (2013), der eine Version des Modells nach Melitz (2013) zugrundelegt, liegen sie zwischen 18 und 50 Prozent. Das größere Land erhebt den höheren Zoll, und der Wohlfahrtsschaden liegt zwischen 2 und 8 Prozent der gesamtwirtschaftlichen Wohlfahrt der Situation ohne Zölle. Die Reaktionsfunktionen sind negativ geneigt: Der optimale Zoll sinkt in der Zollhöhe des Handelspartners. Zölle sind also strategische Substitute. Ossa (2011) verwendet ein etwas simpleres Modell ohne Firmenheterogenität, weitet den Blick aber für eine Vielzahl von Wirtschaftszweigen und Ländern. Hier liegen die Nash-Zölle noch etwas höher, bei knapp 60 Prozent. Fast ein Viertel der Handelsgewinne ginge verloren.

\subsection{Sind Handelskriege zu gewinnen?}

In der älteren theoretischen Literatur beschäftigte man sich gerne mit der Frage, ob es überhaupt möglich ist, einen Handelskrieg zu gewinnen - dass sich also ein Land in einem nicht-kooperativen Gleichgewicht besser stellt als in einer Situation, in der alle Länder auf Zölle verzichten. Kennan und Riezman (1988) zeigen, dass sehr große Länder im nicht-kooperativen (Nash-)Gleichgewicht unter Umständen gewinnen können. In komplexeren Modellen mit vielen Ländern und Wirtschaftszweigen kann es durchaus passieren, dass sich einzelne Länder in einer Situation, in der Nash-Zölle gesetzt werden, besser stellen als in einem kooperativen Gleichgewicht. Das geschieht beispielsweise, wenn der Handelskrieg die Terms of trade eines kleinen, sehr stark spezialisierten Landes deutlich verbessert. Aber die Welt insgesamt ist erheblich schlechter gestellt. Vor allem kleinere Länder verlieren; ihnen fallen schließlich auch die größten Vorteile durch Teilnahme an der internationalen Arbeitsteilung zu. Und meine vielen eigenen Versuche, mit dem realistisch kalibrierten Ifo-Handelsmodell Situationen $\mathrm{zu}$ generieren, in denen sich die Vereinigten Staaten - als die größte Volkswirtschaft der Welt - durch nicht-kooperatives Verhalten besser stellen, sind allesamt gescheitert. Selbst die Vereinigten Staaten sind deutlich zu klein. Nach Felbermayr et al. (2013) kann auch ein Land, das (fast) die ganze Wirtschaftskraft auf sich vereint, den Handelskrieg nicht gewinnen.

In der Praxis ist das multilaterale System der beste Schutz gegen Erpressungsversuche großer Länder. Ein wichtiger Vorteil der Welthandelsorganisation (WTO) und ihres Vorläufers, des Allgemeinen Abkommens über Zölle und Handel (GATT), besteht darin, dass auch das größte Mitglied im Vergleich zu einer Koalition der anderen Län- der ein kleines Land ist, so dass es kaum möglich erscheint, Handelskriege zu gewinnen, solange die anderen Mitglieder ein Mindestmaß an Einigkeit zustande bringen. Das Kennan-Riezman-Resultat scheint also nicht viel mehr zu sein als eine theoretische Möglichkeit mit begrenzter empirischer Relevanz. ${ }^{8}$

Eines wird in der Literatur deutlich: „Handelsgewinne sind groß und Handelskriege teuer“, so fasst Ossa (2018) die Literatur zusammen. Dabei ist noch in Betracht zu ziehen, dass man mit den üblichen quantitativen Handelsmodellen die Handelsgewinne typischerweise deutlich unterschätzt, was an der statischen Natur der Modelle und der daraus folgenden Missachtung dynamischer Handelsgewinne liegt. Daher werden auch die Kosten von Handelskriegen unterschätzt. Umgekehrt gilt: Die handelspolitische Kooperation, die wir seit dem Zweiten Weltkrieg erlebt haben, hat erhebliche Wohlfahrtsgewinne ermöglicht.

\subsection{Der überraschende Erfolg von GATT und WTO}

Das multilaterale Handelssystem verdankt seine Existenz der Einsicht, dass nicht-kooperatives handelspolitisches Verhalten der Länder wirtschaftliche Schäden nach sich zieht. GATT und WTO bieten ein Forum, auf dem die Regierungen erfolgreich über die Senkung von Zöllen verhandeln und Handelskonflikte beilegen können.

Im historischen Vergleich liegen die Durchschnittszölle gegenwärtig auf einem sehr niedrigen Niveau. ${ }^{9}$ In der Wirtschaftskrise der dreißiger Jahre war beispielsweise der Durchschnittszoll der Vereinigten Staaten mehr als vierzehnmal so hoch wie der heutige. Der globale (ungewichtete) Durchschnittszollsatz ist seit dem Ende des Zweiten

8 Ob ein Land einen Handelsbilanzüberschuss oder ein -defizit hat, spielt für die Frage, ob es einen Handelskrieg gewinnen kann, insofern eine Rolle, als die Höhe der Importe die Möglichkeit von Vergeltungszöllen beschränkt; siehe ausführlicher dazu weiter unten. 9 Die Quantifizierung des Grades der Protektion einer Volkswirtschaft ist nicht trivial. Typischerweise existieren unterschiedlich hohe Zölle für mehrere tausend Produkte. Die Zölle sind nicht immer in Ad-valorem-Form festgelegt, sondern können auch feste Beträge sein; sie sind oft als Quotenzölle organisiert (das heißt, ihre Höhe ist abhängig vom Handelsvolumen). Selbst wenn man Ad-valorem-Zölle für alle Produkte hätte, bliebe die Aggregation eine Herausforderung. Wenn man mit Handelsvolumina gewichtet, verzerrt dies den Durchschnittszoll nach unten, weil gerade Produkte mit sehr hohen Zöllen wenig gehandelt werden. Wenn man ungewichtete Durchschnitte berechnet, kommt es ebenfalls zu einem Messfehler, weil Güter in die Durchschnittsberechnung eingehen, die auch bei einem Zollsatz von Null gar nicht importiert werden. 
Weltkriegs von ungefähr 40 auf 4 Prozent gefallen (Bagwell und Staiger 2002). Das ist eine sehr beachtliche Erfolgsgeschichte, ohne die der rasche wirtschaftliche Aufstieg Deutschlands nach dem Krieg oder der wirtschaftliche Siegeszug Ostasiens kaum denkbar gewesen wären (Baldwin 2016). Die Erfolgsgeschichte von GATT und WTO ist insofern auch überraschend, als sie ohne eigentliche Rechtsdurchsetzung auskommt. Wenn Länder gegen Recht verstoßen und die zuständigen Gremien dies feststellen, gibt es keinen Weg, das abweichende Land zu einer Rückkehr zum Recht zu zwingen.

Tabelle 1: Höhe von Zöllen und Budgetanteile von Zolleinnahmen

\begin{tabular}{|c|c|c|c|c|}
\hline \multirow[b]{3}{*}{ EU } & \multicolumn{2}{|c|}{1990} & \multicolumn{2}{|c|}{2015} \\
\hline & \multicolumn{2}{|c|}{ Zollsatz Budgetanteil } & \multicolumn{2}{|c|}{ Zollsatz Budgetanteil } \\
\hline & $6,0 \%$ & $26,1 \%$ & $3,0 \%$ & $13,6 \%$ \\
\hline USA & $4,7 \%$ & $2,7 \%$ & $2,8 \%$ & $1,9 \%$ \\
\hline Japan & $4,4 \%$ & $1,4 \%$ & $2,8 \%$ & $1,6 \%$ \\
\hline Ägypten** & $17,1 \%$ & $24,0 \%$ & $10,5 \%$ & $6,8 \%$ \\
\hline Brasilien & $19,0 \%$ & $3,9 \%$ & $10,3 \%$ & $5,1 \%$ \\
\hline China* & $14,7 \%$ & $6,7 \%$ & $5,6 \%$ & $4,0 \%$ \\
\hline Indien & $56,6 \%$ & $35,8 \%$ & $7,5 \%$ & $15,2 \%$ \\
\hline 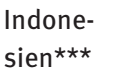 & $15,1 \%$ & $6,6 \%$ & $5,4 \%$ & $2,5 \%$ \\
\hline \multicolumn{5}{|c|}{$\begin{array}{l}\text { Quelle: Weltbank } \\
\text { Anmerkung: Meistbegünstigungszölle, gewichtete Durchschnitte, } \\
\text { alle Produkte. * statt 1990: 2000; }{ }^{\star *} \text { statt 1990: 1995; }{ }^{\star * *} \text { statt 2015: } \\
\text { 2016. Die Budgetanteile beziehen sich auf die Gesamtbudgets der } \\
\text { Staaten (EU: Zölle und Importabgaben im Verhältnis zu den } \\
\text { gesamten Eigenmitteln). }\end{array}$} \\
\hline
\end{tabular}

Tabelle 1 zeigt die Entwicklung der zurückliegenden 25 Jahre. Die WTO nahm 1995 ihre Arbeit auf; 2001 trat ihr China bei. Der von der EU erhobene durchschnittliche handelsgewichtete Meistbegünstigungszoll (jener Zoll, der gegenüber WTO-Mitgliedern maximal angewandt werden darf, mit denen kein Freihandelsabkommen besteht) lag 2015 bei 3 Prozent; 1990 war er noch doppelt so hoch. Der europäische Zollsatz liegt damit leicht über dem amerikanischen (2,8 Prozent), ist aber seit 1990 deutlich stärker gefallen. ${ }^{10}$ Ähnliches gilt für Japan. Schwellenländer wie Ägypten oder Brasilien erheben deutlich höhere Zölle; Chinas Durchschnittszoll ist mit 5,6 Prozent doppelt so hoch wie jener, den die Vereinigten Staaten oder die EU

10 Wenn man ungewichtete Durchschnitte betrachtet, erscheint die EU noch deutlich stärker protektionistisch als die Vereinigten Staaten (5,2 versus 3,5 Prozent; siehe Felbermayr 2018). erheben. Gleichwohl ist auch dieser Zollsatz historisch gering. Indien hatte 1990 noch einen Durchschnittszoll von 57 Prozent, heute liegt er bei 7,5 Prozent.

Die Bedeutung von Zöllen in den staatlichen Haushalten ist in den allermeisten Ländern deutlich geschrumpft. In der EU machten Zölle (und andere Handelsabgaben) 1990 noch ungefähr 26 Prozent der gesamten Eigenmittel aus; heute liegt der Anteil bei 14 Prozent. In den Vereinigten Staaten und Japan liegt der Budgetanteil bei weniger als 2 Prozent. Wenn man das Budget der EU mit den Budgets ihrer Mitglieder konsolidierte, käme man auf eine ähnlich geringe Größe. In Schwellen- und Entwicklungsländern ist die Bedeutung von Zöllen für die Budgets noch höher. ${ }^{11}$

\subsection{Die positive Wirkung von Reziprozität}

Der Erfolg von GATT und WTO wird in der Literatur (Bagwell und Staiger 1999, 2002, 2016) auf das Prinzip der Reziprozität zurückgeführt und darauf, dass die Regeln eingehalten wurden, obwohl es keine Organe der Rechtsdurchsetzung gibt. Das Prinzip der Reziprozität beruht auf der Überlegung, dass Länder dann bereit sind, Zölle aufzugeben, wenn sich dabei ihre Terms of trade nicht verschlechtern. Das ist dann nicht der Fall, wenn die „Konzessionen“, wie Zollsenkungen in der WTO-Sprache heißen, so ausfallen, dass die Verhandlungspartner vergleichbare Zuwächse in ihren Exporten erwarten können. Dann verändert sich weder das relative Angebot ihrer Güter noch die relative Nachfrage danach, und die Terms of trade (der relative Preis der Exportgüter im Vergleich zu den Importgütern) bleiben ebenfalls konstant.

Das Prinzip der Reziprozität und die damit verbundenen Begrifflichkeiten mögen recht merkantilistisch klingen. In der Tat läge es im Interesse jedes einzelnen kleinen Landes, seine Zölle auf Null zu senken, auch wenn die Gegenseite dies nicht tut. Wie schon gezeigt, ist die Annahme des kleinen Landes jedoch weitgehend Fiktion - und damit ist das Prinzip der Reziprozität gerade jener Hebel, mit dem die Länder dem Gefangendilemma entfliehen können.

Ein weiteres sehr wichtiges Prinzip der WTO ist die Meistbegünstigung. Sie stellt sicher, dass die WTO-Mitglieder gegenüber allen anderen Mitgliedern die gleichen Zölle anwenden. Die Meistbegünstigung ist relevant, weil es

11 Brasilien ist interessant: Hier ist der Durchschnittszoll um fast 50 Prozent gefallen, und doch hat sich der Anteil der Zolleinnahmen im Gesamthaushalt erhöht. Die Ursache dafür ist, dass der Handel sehr stark gewachsen ist. 
in der Realität natürlich immer mehr als nur zwei Spieler gibt. Sie gibt kleinen Ländern mehr Macht, als diese sonst hätten. Man würde die Optimalzölle ohne Meistbegünstigung gegenüber großen Ländern klein und gegenüber kleinen Ländern groß wählen. Die Meistbegünstigung wird allerdings durch bilaterale Freihandelsabkommen aller Art und durch spezielle Zollpräferenzen gegenüber Entwicklungsländern untergraben.

\subsection{Tit for tat in der WTO}

Reziprozität ist auch für die Durchsetzung der rechtlichen Ordnung von GATT und WTO entscheidend. Hier gibt es die Schwierigkeit, dass Länder Zollzugeständnisse machen könnten, um bei nächster Gelegenheit gleich wieder von ihren Zusagen zurückzutreten. Weil es keine internationale Handelspolizei gibt, die Regelverstöße bestrafen könnte, muss das System so ausgestaltet sein, dass die Spieler aus eigenem Interesse die Spielregeln einhalten. Bagwell und Staiger (2002) zeigen, dass man die konstituierenden Texte von GATT und WTO als Kodifizierung sogenannter „Supergame“-Strategien verstehen kann - als Strategien, die den Spielern in wiederholten Spielen zur Verfügung stehen. Das multilaterale System gibt den Ländern ein Protokoll in die Hände, das sie durchlaufen dürfen, wenn ein Partner die Regeln bricht. Konkret heißt dies, dass sie Vergeltungsmaßnahmen einsetzen dürfen, die streng reguliert sind und die Neutralisierung der Terms-oftrade-Effekte zum Ziel haben. In den WTO-Texten ist in diesem Zusammenhang sinnigerweise von einem „Balancing“ die Rede, einem Ausgleich. Vergeltungszölle dürfen zu keinen größeren Zolleinnahmen führen als die Zölle des abweichenden Landes. Im WTO-Recht ist daher auch nirgendwo die Rede von Strafzöllen. Es geht immer nur um die Wiederherstellung des Anfangszustandes, um Kompensation, nicht aber um eine darüber hinausgehende Bestrafung einer Regierung.

Es bleibt jedem Land im Einzelnen überlassen, auf die Importe welcher Güter es die Vergeltungszölle erhebt. Die Ausnutzung dieser Freiheit erlaubt dann doch in gewisser Weise eine „Bestrafung“ der abweichenden Regierung, weil man bewusst Güter ins Visier nehmen kann, bei denen der höchste politische Schaden für die abweichende Regierung zu erwarten ist.

\subsection{Vergeltungszölle und die Stabilität kooperativer Gleichgewichte}

Die im WTO-Recht vorgesehenen Vergeltungszölle sind sinnvoll; sie sind für die Stabilität eines kooperativen Gleichgewichtes konstitutiv. Wenn ein Land A einen höheren als den vereinbarten Zollsatz anwendet, kann es damit die eigene aggregierte Wohlfahrt erhöhen, falls der nun angewandte Zoll näher am unilateralen Optimalzoll liegt als der vorherige. Dabei schadet es allerdings seinen Handelspartnern, weil sich deren Terms of trade verschlechtern. Wenn die Handelspartner nun ihrerseits (Vergeltungs- oder Retorsions-)Zölle setzen, die die Terms of trade wieder in den Ausgangszustand zurückversetzen, dann verhindern sie, dass sich das abweichende Land A auf ihre Kosten bereichert. Alle Länder verzeichnen nun Wohlfahrtsverluste, aber die Verluste in den ursprünglich ,angegriffenen“ Ländern fallen geringer aus, als wenn es die Terms-of-Trade-Verschlechterung einfach hingenommen hätte. ${ }^{12}$

Ein solches Tit-for-Tat-Verhalten kann viele Gleichgewichte hervorbringen, wie das Folk-Theorem wiederholter Spiele besagt, aber Kooperation kann dazu gehören (Holler und Illing 2005). Voraussetzung ist, dass es in der Zukunft auch tatsächlich noch weitere Interaktionen (Spielzüge) gibt und dass die Zukunft in den Nutzenfunktionen der Spieler ein positives Gewicht hat. Im Kontext von Handelspolitik heißt dies, dass populistische Regierungen, denen es nur um sehr kurzfristige Erfolge geht, eher von kooperativem Verhalten abweichen als solche, die sich einer längeren politischen Tradition verpflichtet fühlen.

Wenn die Möglichkeit von Vergeltungsmaßnahmen glaubhaft ist, dann sollten Handelskonflikte erst gar nicht entstehen, weil die Spieler nicht von der kooperativen Strategie abweichen. Die beste Drohung ist jene, der keine Taten folgen müssen. In der Wirklichkeit herrscht aber ein erhebliches Ausmaß an Unsicherheit über die Motive der Spieler, über ihre innenpolitische Durchsetzungskraft oder über die Stärke ihrer Zukunftspräferenzen. Es kann also geschehen, dass die Regierung eines Landes die Entschlossenheit der Handelspartner testet, um herauszufinden, ob es tatsächlich Vergeltungsmaßnahmen fürchten muss.

Im Übrigen ist es gänzlich irrelevant, ob die abweichenden Zölle des Landes A Optimalzölle in dem Sinne

12 In einer Situation mit vielen heterogenen Ländern, die unterschiedlich von den neuen Zöllen betroffen sind (und von diesen sogar profitieren können, wenn sie Nettoimporteure der von Land A verzollten Güter sind), ist die Situation theoretisch nicht mehr eindeutig. 
sind, dass Land A damit die Wohlfahrt maximieren möchte, und ob die Vergeltungszölle der Handelspartner ein Nash-Gleichgewicht bilden. Aufgabe der Vergeltungszölle ist es sicherzustellen, dass nicht-kooperatives Verhalten erfolglos bleibt. Zentral ist hingegen, dass die Länder glaubhaft mit Retorsion drohen können. Dazu müssen sie über die entsprechenden innenpolitischen Voraussetzungen verfügen. Das ist nicht immer unproblematisch. Denn Vergeltungszölle lindern zwar die gesamtwirtschaftlichen Schäden der Abweichung von Land A, aber sie bewirken Umverteilungseffekte, und die Erwartung solcher Effekte setzt politökonomische Mechanismen in Kraft, die eine Regierung zum Verzicht auf Retorsion bewegen können.

Manchmal ist zu hören, eine WTO-konsistente Vergeltung sei nur in dem Ausmaß möglich, in dem ein Land tatsächlich von dem abweichenden Land importiere. Das ist richtig. Wenn Land A beispielsweise im großen Stil aus Land B importiert, dorthin aber nur sehr wenig exportiert, dann kann eine Tit-for-Tat-Strategie nicht erfolgreich sein. Solche extremen Situationen sind zwischen großen Volkswirtschaften allerdings selten, und sie können WTO-rechtlich dadurch kompensiert werden, dass die Höhe des Vergeltungszollsatzes jene des ursprünglich von Land A eingesetzten Zolles übersteigt. Dazu gibt es die Möglichkeit sogenannter asymmetrischer Vergeltung. Damit betritt man zwar eine handelsrechtliche Grauzone, aber man kann sich als Antwort auf güterwirtschaftliche Zölle durchaus Maßnahmen im Dienstleistungshandel vorstellen, der ebenfalls im WTO-Recht geregelt ist. Wenn man den Wirkungsbereich der WTO verlässt, eröffnen sich noch viele weitere Retorsionsmöglichkeiten, zum Beispiel in der Wechselkurspolitik, in der Kapitalverkehrspolitik, in der Regulierung ausländischer Konzerne und so weiter.

Es mag paradox erscheinen, dass mit Vergeltungszöllen ein liberales Freihandelssystem verteidigt werden soll. Aber wenn die Spieler davon ausgehen können, dass es im Fall einer Abweichung zu keiner Reaktion der Handelspartner kommt, dann gibt es keine Gründe, warum sie nicht ihre dominante Strategie der Nicht-Kooperation spielen sollten. Mit anderen Worten: Die Drohung mit Vergeltung und die tatsächliche Vergeltung im Ernstfall stabilisieren das kooperative Gleichgewicht. Vornehme Zurückhaltung könnte hingegen den Zusammenbruch der multilateralen Ordnung herbeiführen.

Grundprinzip dieser Überlegungen ist, dass die Regierungen der Länder ihren eigenen Vorteil suchen, dass sie sozusagen eine kurzfristige „My-country-first“-Politik verfolgen und nicht altruistisch im Sinne der Verfolgung eines höheren Zieles der Staatengemeinschaft handeln, von dem sie selber langfristig profitiert. Solche Regierungen weichen vom kooperativen Verhalten ab, wenn sie erwarten dürfen, dies ungestraft tun zu können. Sonntagsreden und Appelle an eine globale Verantwortung können die Drohung mit Vergeltung nicht ersetzen, solange sich politischer Erfolg vor allem am Erreichen nationaler Ziele bemisst.

\section{Die Vereinigten Staaten als handelspolitischer Aggressor?}

\subsection{Die WTO steht vor echten Problemen}

Die letzte große Reform des multilateralen Systems, die zwischen 1986 und 1994 in der sogenannten UruguayRunde verhandelt wurde und mit der Gründung der WTO endete, ging zentral davon aus, dass sich das demokratisch-marktwirtschaftliche System auf einem unaufhaltsamen Siegeszug befinde und dass nach dem Ende des Kommunismus in Osteuropa auch andere autokratische und planwirtschaftliche Regimes verschwänden. Das System ist darauf angelegt, dass die maßgeblichen Mitglieder jedenfalls dem Grundsatz nach Marktwirtschaften sind. Eine zweite wichtige Annahme bei der Schaffung der WTO war, dass die globalen Kräfteverhältnisse noch viele Jahrzehnte stabil bleiben. Beide Hypothesen falsifiziert vor allem die Volksrepublik China, die seit 2001 Vollmitglied der WTO ist. Ihr Aufstieg und ihre kompromisslose Industrie- und Technologiepolitik waren in der WTO-Philosophie nicht vorgesehen.

Die WTO verbietet zwar Exportsubventionen relativ rigoros; sie hat aber wenig Handhabe gegen Subventionen allgemeiner Art, zum Beispiel in Form einer Unterstützung maroder Unternehmen durch staatliche Banken. Es gibt auf multilateraler Ebene kein Analogon zum Beihilfenrecht der Europäischen Union. Allerdings gäbe es hier auch riesige Widerstände: $\mathrm{Zu}$ viele WTO-Mitglieder, die EU miteingeschlossen, verteilen hohe Subventionen an viele Wirtschaftsbranchen, von Hilfen an die Landwirtschaft bis hin zur Förderung grüner Energien.

Die WTO leidet aber noch an einem weiteren grundlegenden Problem, das für die Diskussion der Reziprozität sehr relevant ist. Die Uruguay-Runde hat eine recht asymmetrische Zollsituation hervorgebracht, siehe Tabelle 1. Ein weiterer Abbau dieser Zölle gemäß dem Reziprozitätsprinzip ist daher nicht oder nur schwer möglich. Im Rahmen eines reinen Zollabkommens können die Vereinigten Staaten, die EU und Japan im Prinzip kaum noch $\mathrm{Zu}$ geständnisse machen, weil ihre Zölle schon sehr niedrig sind. Für weitere Fortschritte ist daher die Kombination von Zollverhandlungen mit nicht-tarifären Themen erfor- 
derlich. Dies hat sich aber als extrem schwierig herausgestellt. Der Widerstand in den entwickelten Ländern gegen den Abbau solcher Barrieren, die meist regulatorischer Natur sind, ist erheblich.

\subsection{Die neue amerikanische Handelspolitik}

Mit der Wahl von Donald Trump zum Präsidenten der Vereinigten Staaten am 8. November 2016 begann eine neue Ära in der internationalen Handelspolitik. Die neue Ära hat viele Facetten; eine davon ist der starke Fokus allen politischen Handelns auf die Leistungsbilanz. Schon 1990 sagte Trump in seinem Interview mit dem Playboy-Magazin, dass seine erste Amtshandlung, wenn er Präsident würde, „a tax on every Mercedes-Benz rolling into this country and on all Japanese products“ wäre. Das größte amerikanische Problem sei das hohe Handelsdefizit. Diese Geisteshaltung hat sich nicht verändert. Immer wieder klagt Trump, andere Länder übervorteilten die Vereinigten Staaten. In seinem Lamento über das globale Handelssystem tauchen immer wieder die durchaus kritikwürdigen Schwächen der WTO auf; wobei Trump das amerikanische Leistungsbilanzdefizit offenbar als Beweis dafür versteht, dass die Vereinigten Staaten benachteiligt würden.

Tabelle 2: Salden der amerikanischen Leistungsbilanz nach Handelspartnern und Transaktionsarten, in Mrd. Dollar

\begin{tabular}{lrrrrr}
\hline & Güter & $\begin{array}{r}\text { Dienst- } \\
\text { leistungen }\end{array}$ & \multicolumn{2}{c}{$\begin{array}{c}\text { Primär- } \\
\text { einkommen }\end{array}$} & $\begin{array}{r}\text { Sekundär- Leistungs- } \\
\text { bilanz }\end{array}$ \\
\hline mit China & -376 & 38 & -17 & -4 & -358 \\
\hline $\begin{array}{l}\text { mit } \\
\text { Mexiko }\end{array}$ & -76 & 7 & -1 & -16 & -86 \\
\hline mit Japan & -70 & 14 & -26 & 3 & -80 \\
\hline mit Korea & -23 & 12 & 0 & -2 & -12 \\
\hline mit & -23 & 26 & 14 & -3 & 14 \\
Kanada & & & & & \\
\hline mit EU & -153 & 51 & 106 & 10 & 14 \\
\hline $\begin{array}{l}\text { mit Rest } \\
\text { der Welt }\end{array}$ & -91 & 94 & 141 & -102 & 42 \\
\hline $\begin{array}{l}\text { USA } \\
\text { gesamt }\end{array}$ & -811 & 243 & 217 & -115 & -466 \\
\hline
\end{tabular}

Quelle: US Bureau of Economic Analysis

Anmerkung: Primäreinkommen enthalten Einkommen aus Portfoliound Direktinvestitionen im Ausland. Sekundäreinkommen sind Transfers ohne erkennbare Gegenleistung (zum Beispiel Überweisungen von Migranten in ihre Herkunftsländer, Zahlungen an internationale Organisationen).
Dabei geht es dem Präsidenten vor allem um den Güterhandel. Hier betrug 2017 das Defizit der Vereinigten Staaten 811 Milliarden Dollar (4,2 Prozent des BIP) und wird wohl 2018 noch etwas größer ausfallen. Tabelle 2 zeigt, dass die Vereinigten Staaten mit allen maßgeblichen Handelspartnern Defizite haben; die größten mit China (376 Milliarden), mit der EU (153 Milliarden), Mexiko (76 Milliarden) und Japan (70 Milliarden). Gleichzeitig haben die Vereinigten Staaten einen Überschuss im Dienstleistungshandel mit den meisten Ländern, der sich auf 243 Milliarden Dollar addiert (1,3 Prozent des BIP). Ganz offensichtlich verfügt Amerika über einen komparativen Vorteil im Handel mit Dienstleistungen und über einen komparativen Nachteil im Handel mit Gütern.

Das Land erzielte 2017 auch einen deutlichen Überschuss der Primäreinkommen: Amerikanische Kapitaleinkommen im Ausland übersteigen die Kapitaleinkommen der Ausländer in den Vereinigten Staaten um 217 Milliarden Dollar. In der Bilanz der Sekundäreinkommen haben die Vereinigten Staaten ein Defizit. Hier schlagen sich zum Beispiel die Überweisungen von Migranten in ihre Heimatländer nieder, Zahlungen an amerikanische Militärs im Ausland, Entwicklungshilfe, Strafzahlungen von amerikanischen Konzernen an die EU-Wettbewerbsbehörden oder auch Zahlungen an internationale Organisationen.

Die wichtigste Botschaft der Tabelle aber ist, dass die Vereinigten Staaten zwar mit allen maßgeblichen Ländern Defizite im Güterhandel haben, die für die gesamtwirtschaftliche Wohlfahrt relevante Leistungsbilanz aber mit Kanada und der EU relativ ausgeglichen ist. ${ }^{13}$ Diese Länder verfügen daher auch über erhebliche Möglichkeiten einer asymmetrischen Vergeltung.

\subsection{Rückkehr blanker Machtpolitik}

Das Leistungsbilanzdefizit der Vereinigten Staaten hat, der Meinung der gegenwärtigen amerikanischen Regierung zufolge, vor allem handelspolitische Ursachen..$^{14}$ Amerikanische Unternehmen hätten keinen fairen Zugang zu ausländischen Märkten, während der amerikanische Markt offen sei; ausländische Unternehmen würden direkt und

13 EUROSTAT berichtet von einem Leistungsbilanzüberschuss der EU gegenüber den Vereinigten Staaten. Allerdings scheinen die europäischen Daten nicht richtig zu sein; siehe Braml und Felbermayr 2018.

14 Siehe beispielsweise in der 2017 Trade Policy Agenda des amerikanischen Präsidenten; online verfügbar unter https://ustr.gov/site s/default/files/files/reports/2017/AnnualReport/AnnualReport2017.p df 
indirekt subventioniert und verschafften sich damit unfaire Wettbewerbsvorteile; die Vereinigten Staaten würden durch internationale Vereinbarungen übervorteilt und ihrer Souveränität beraubt. Der amerikanische Präsident möchte all diese vermeintlichen Probleme beheben; dafür brauche es vor allem „Toughness of attitude“, sagte er 1990 dem Playboy Magazine.

Im 19. Jahrhundert zwangen die Weltmächte Länder wie China oder Japan mit militärischen Mitteln, ihre Grenzen zu öffnen. Nach dem Zweiten Weltkrieg und dem Ende des Kolonialismus kamen handelspolitische Vereinbarungen auf dem Verhandlungsweg zustande, wobei die Regeln von GATT/WTO und allgemeinem Völkerrecht galten. Der Einsatz blanker Macht zur Durchsetzung wirtschaftlicher Interessen verschwand nicht, er wurde aber weitgehend in Verhandlungsräume verbannt und es hat sich ein kooperativer Umgangston etabliert, der Vertrauen und konstruktives Verhalten fördert.

Trump hält von diesen Mitteln offenbar wenig. Er setzt seinen Trumpf - die Größe des amerikanischen Marktes mit aller Macht ein. Das haben die Vorgängerregierungen nicht getan, und darum geht er mit deren Handelsabkommen hart ins Gericht. Seine erste Amtshandlung bestand darin, den politischen Prozess der Ratifikation des Transpazifischen Partnerschaftsabkommens (TPP) zu beenden, das die Regierung von Präsident Barack Obama mit 11 Pazifikanrainerstaaten verhandelt hatte, mit dem Ziel einer wirtschaftlichen Eindämmung Chinas. Auch von dem ebenfalls in der Amtszeit Obamas geplanten Abkommen mit der EU, TTIP, scheint er bis jetzt nicht viel zu halten.

Trump will bestehende bilaterale Abkommen neu verhandeln und schlägt dabei eine aggressive Linie ein, mit dem Ziel, die eigene Marktmacht $\mathrm{zu}$ hebeln und $\mathrm{Zu}$ geständnisse zu erzwingen. Die Regeln der WTO hindern den Präsidenten bei der Ausnutzung der amerikanischen Größe, weil er bei der Neuverhandlung von Vereinbarungen immer damit rechnen muss, einen sehr erheblichen Anteil der anderen 163 WTO-Mitglieder gegen sich zu haben. Es ist daher nur logisch, dass ihm an einer Schwächung des multilateralen Systems liegt und er das Heil in bilateralen „Deals“ sucht. Seine Taktik besteht offenbar darin zu zeigen, dass die Vereinigten Staaten auch bereit wären, sich außerhalb des Rahmens der WTO zu bewegen. Damit baut Trump eine Drohkulisse auf, die ihm erhebliche Verhandlungsmacht verleiht.

Die Ablehnung, die der amerikanische Präsident der WTO entgegenbringt, ist in mehreren Dokumenten beurkundet ${ }^{15}$. Darin kritisiert die aktuelle Regierung der Ver-

15 Quelle siehe die vorhergehende Fußnote. einigten Staaten multilaterale Handelsabkommen und macht klar, dass sie bilaterale Abkommen bevorzugt. Sie rügt die WTO dafür, dass sich ihre Rechtsprechung verselbständigt habe und sich immer mehr von den Intentionen der Gründer entferne. Wenn amerikanische Interessen nicht mit einer fortwährenden Mitgliedschaft in der WTO vereinbar seien, heißt es, dann müssten die Vereinigten Staaten eben austreten.

Im Juli 2018 sind Entwürfe eines „Fair and Reciprocal Trade Act“ (von Kritikern sofort als FART-Act karikiert) durchgesickert. Dieses Gesetz würde den Präsidenten ermächtigen, Zölle in jener Höhe zu erheben, wie es die Handelspartner gegenüber den Vereinigten Staaten tun. Das widerspräche den Verpflichtungen der Vereinigten Staaten aus der Uruguay-Runde. Außerdem könnte der Präsident die Meistbegünstigung umgehen und unterschiedlichen Ländern ganz unterschiedliche Zölle vorschreiben, auch wenn kein formales Handelsabkommen vorliegt. Das Weiße Haus hat sich zwar von FART distanziert, aber das Dokument zeigt doch sehr deutlich, dass es in der Regierung starke Kräfte gibt, die jene Fesseln, welche die WTO den Vereinigten Staaten anlegt, loswerden wollen. Auch die FART-Initiative stärkt am Ende die Verhandlungsmacht des amerikanischen Präsidenten, weil sie zeigt, wie weit er zu gehen bereit wäre.

\subsection{Die vier Felder der Trump'schen Handelspolitik}

Die Trump'sche Handelspolitik hat bisher vier große Konflikte hervorgebracht. Im ersten Konflikt geht es um Waschmaschinen und Solarpaneele. Im Herbst 2017 veröffentlichte die amerikanische Handelsbehörde USTR einen Bericht, dessen Verfasser globale Schutzzölle auf Waschmaschinen und Solarpaneele empfehlen. Die Importe seien so drastisch und unvorhersehbar gestiegen, dass die Tatbestände für solche Schutzmaßnahmen nach Artikel GATT XIX gegeben seien, heißt es darin. Schutzzölle („Safeguard tariffs“) sind WTO-konforme Notmaßnahmen erga omnes, das heißt gegenüber allen Handelspartnern, die zulässig sind, um heimische Unternehmen temporär aus existentiellen Notlagen zu retten. ${ }^{16}$ Das WTORecht sieht aber vor, dass die Handelspartner Kompensation erhalten, indem ihnen Konzessionen in anderen Bereichen angeboten werden. Falls solche Angebote nicht erfolgen, können die Länder nach Ablauf einer gewissen Frist selbst Ausgleichsmaßnahmen definieren.

16 Safeguards müssen dem Meistbegünstigungsgrundsatz genügen. 
Im Januar 2018 traten diese Schutzzölle in Kraft. Sie umfassen Importe von Solarpaneelen im Wertumfang von 8,5 Milliarden Dollar und von Waschmaschinen für 1,8 Milliarden Dollar. Die Maßnahmen treffen Hersteller aus vielen Ländern, auch aus Europa. Es wurde keine Kompensation angeboten. China hat sich mit Anti-DumpingZöllen auf Sorghumhirse aus den Vereinigten Staaten gewehrt; Korea hat vor der WTO geklagt. Im Frühsommer haben die Verhandlungen zwischen China und den Vereinigten Staaten begonnen; China nahm die Sorghumzölle zurück. Die Verhandlungen führten zu nichts. China klagte im August vor der WTO.

Die zweite Auseinandersetzung geht um Stahl und Aluminium. Im April 2017 gab der amerikanische Präsident eine Untersuchung in Auftrag, um zu überprüfen, ob Importe von Stahl und Aluminium die nationale Sicherheit der Vereinigten Staaten gefährden. Das WTO-Recht bietet mit Artikel XXI eine formale Grundlage, die Mitgliedern erlaubt, im Falle einer Sicherheitsbedrohung Konzessionen an einzelne Mitglieder zurückzunehmen. ${ }^{17} \mathrm{Im}$ Unterschied zu den übrigen WTO-Regeln ist die Sicherheitsausnahme nicht justiziabel. Jedem Land bleibt unbenommen, selbst zu definieren, was eine Bedrohung der nationalen Sicherheit darstellt. Kommentatoren bezeichnen daher Artikel XXI als die gleichsam „nukleare Option“ im WTO-Recht. ${ }^{18}$ Bisher gibt es keine Urteile zur Sicherheitsausnahme, denn die „nukleare Option“ wurde bisher kaum gezogen. Dies ist nicht überraschend, denn Länder, die in Friedenszeiten Artikel XXI bemühen, stellen damit das gesamte Regelwerk der WTO in Frage.

Mit der Sicherheitsbegründung hat die amerikanische Regierung maximale Flexibilität in der Ausgestaltung ihrer Handelspolitik. Die Frage ist, ob Importe von Stahl und Aluminium tatsächlich die nationale Sicherheit der Vereinigten Staaten bedrohen oder ob es sich um einen Vorwand handelt. Nach Angaben der amerikanischen Regierung werden rund 30 Prozent des Stahlverbrauchs importiert. ${ }^{19}$ Stahlimporte aus Ländern, die nicht militärisch mit den Vereinigten Staaten kooperieren, wie China, Vietnam oder Russland, machen etwa 10 Prozent der Importe aus. Rund 90 Prozent der Importe kommen von Alliierten.

17 Im Unterschied zu Schutzzöllen unterliegen Maßnahmen nach Artikel XXI nicht dem Meistbegünstigungsgrundsatz.

18 Siehe eine Bloomberg-Meldung, online verfügbar unter https://w ww.bloomberg.com/news/articles/2017-06-28/trump-faces-laugh-tes t-as-he-weighs-nuclear-option-for-steel

19 Siehe die Mitteilung der amerikanischen Regierung, online verfügbar unter https://www.trade.gov/steel/countries/pdfs/import s-us.pdf
Nichtsdestotrotz schlussfolgerte die Behörde USTR im Februar 2018, dass diese Importe tatsächlich die nationale Sicherheit der Vereinigten Staaten bedrohten. Am 1. März kündigte der Präsident Zölle von 25 Prozent auf Stahl- und Stahlprodukte und von 10 Prozent auf Aluminium- und Aluminiumprodukte an, die insgesamt 48 Milliarden Dollar an Importen betreffen. Wenige Tage später kündigte die EU an, gegen solche Zölle mit Vergeltungsmaßnahmen zu reagieren. Sie stellte die berühmte Juncker-Liste vor, die neben Stahl- und Aluprodukten auch Erdnussbutter, Whiskey, Motorräder und Orangensaft umfasst. Die EUKommission argumentierte schlüssig, die im Bericht der USTR gebotenen Argumente stellten eher eine Begründung für Schutzzölle gemäß Art. XIX GATT dar als eine genuine sicherheitspolitische Bedrohung; sie besitze daher ein Recht auf Kompensation. Die Vereinigten Staaten nahmen die EU daraufhin vorläufig von der Anwendung der Zölle aus. Es begannen Verhandlungen über andere Maßnahmen, mit dem Ziel einer Verringerung der europäischen Exporte in die Vereinigten Staaten. Es kam zu keiner Einigung, und am 1. Juni traten die Zölle auch gegenüber der EU in Kraft. Drei Wochen später wurde die JunckerListe in Kraft gesetzt und es kam auf amerikanische Exporte in die EU im Umfang von 3,2 Milliarden Dollar zu Vergeltungszöllen. ${ }^{20}$ Auch Kanada, China, Mexiko und die Türkei griffen zu Vergeltungszöllen. Die Vereinigten Staaten bestritten deren Rechtmäßigkeit und verklagten diese Länder wenig später.

Der dritte Konflikt handelt von Autos. Am 23. Mai 2018 begann die USTR mit einer Untersuchung, ob Autoimporte ebenfalls die nationale Sicherheit der Vereinigten Staaten bedrohten. Zum Zeitpunkt der Abfassung dieser Zeilen lag noch kein Bericht vor. Die großen Automobilexporteure wie die EU, Korea und Japan waren beunruhigt. Am 25. Juli trafen sich der EU-Kommissionspräsident Jean-Claude Juncker und der amerikanische Präsident Donald Trump in Washington. Sie vereinbarten, Verhandlungen über den Abbau von Handelsbarrieren im transatlantischen Bereich aufzunehmen. Die Vereinigten Staaten verzichteten vorerst auf weitere Zölle, auch in der Automobilindustrie.

Der vierte handelspolitische Konflikt betrifft China, dem die USTR in einer Untersuchung vom 22. März 2018 unfaire Handelspraktiken vorgeworfen hat. Es geht um erzwungenen Technologietransfer und ungenügenden Schutz geistigen Eigentums. Trump hat Ausgleichszölle auf Importe im Ausmaß von 60 Milliarden Dollar angekündigt.

20 Die Reaktion der EU-Kommission ist insofern moderat, als die Gegenzölle nur Importe im Ausmaß von etwa der Hälfte der von den amerikanischen Zöllen betroffenen Exporte ausmachen. 
Wenig später veröffentlichte die amerikanische Regierung eine Liste von 1.333 Produkten (vor allem Technologieprodukte), die mit einem 25-prozentigen Zollsatz belegt werden sollten. Die betroffenen Importe erreichten ein Volumen von 46,2 Milliarden Dollar. China konterte mit einer eigenen Liste von 106 Produkten (Transportgüter und Agrarerzeugnisse), die Importe im Umfang von 50 Milliarden Dollar betrafen. Beide Listen wurden noch verfeinert, und beide Seiten setzten die Zölle am 6. Juli 2018 auf den ersten Teil der Listen (34 Milliarden Dollar) in Kraft. Ende August wurden weitere Volumina von insgesamt 16 Milliarden Dollar beiderseitig mit Zöllen belegt. Im Verlauf des Sommers folgte eine weitere Eskalation: Trump erwog Zölle von 25 Prozent auf weitere 200 Milliarden Dollar chinesischer Exporte in die Vereinigten Staaten.

\subsection{Erste Erfolge Trump'scher Politik?}

Trump hat das nordamerikanische Freihandelsabkommen zwischen den Vereinigten Staaten, Mexiko und Kanada (NAFTA), das unter Präsident Bill Clinton am 1. Januar 1994 in Kraft getreten war, in einer Twitter-Nachricht vom 27. August 2017 als „worst deal ever“ bezeichnet. Die drei Länder befinden sich derzeit in einer Phase der Neuverhandlung des Abkommens, wobei der amerikanische Präsident mehrmals mit dem Ende des Freihandels zwischen den Vereinigten Staaten, Kanada und Mexiko gedroht hat. Mittlerweile bahnt sich ein neues Abkommen, jedenfalls mit Mexiko an, in dem vor allem die sogenannten Ursprungsregeln verschärft werden. Damit wird der regionale Wertschöpfungsanteil, der für zollfreien Handel zwischen den Vereinigten Staaten und Mexiko nachzuweisen ist, von bisher 62,5 auf 75 Prozent erhöht. Europäische und japanische Hersteller, die in Mexiko für den amerikanischen Markt Autos produzieren, werden ihre Wertschöpfungsketten anpassen müssen. Das verringert die Wettbewerbsfähigkeit der mexikanischen Produktion. Vermutlich hätte Mexiko einer solchen Änderung nicht zugestimmt, wenn Trump nicht glaubhaft mit dem kompletten Ende von NAFTA gedroht hätte.

Auch die bereits erfolgte Revision des Abkommens mit Südkorea, das er bei seiner Nominierungsrede im Jahr 2016 als „Job-killing deal“ beschrieben hat (Washington Post vom 12. August 2016), kann nur als eine einseitige Verschiebung zugunsten der Vereinigten Staaten verstanden werden. So wurde die Anzahl von Autos, die die Vereinigten Staaten ohne Nachweis koreanischer Standards nach Korea exportieren dürfen, von 25.000 auf $50.000 \mathrm{im} \mathrm{Jahr}$ angehoben und eine Quote auf Stahl- und Aluminiumexporte Koreas eingeführt.

\section{Der Handelskonflikt zwischen den Vereinigten Staaten und der EU: Versuch einer Bewertung}

Die Verhängung von Stahl- und Aluzöllen gegen NATOVerbündete wegen einer angeblichen Bedrohung der nationalen Sicherheit ist grotesk und tritt WTO-Recht mit Füßen. Es handelt sich vielleicht um die offensichtlichste und am weitesten reichende Regelverletzung, die es in der erfolgreichen Geschichte von GATT und WTO bisher gegeben hat - und das durch das wirtschaftlich und politisch stärkste Land der Welt.

Die Zölle verletzen wirtschaftliche Interessen der EU, auch wenn das Volumen der betroffenen Importe relativ zum BIP der EU klein ist und der volkswirtschaftliche Schaden nur minimal ausfällt. ${ }^{21}$ Die Wohlfahrt Deutschlands, der EU und der anderen WTO-Mitglieder ist aber nicht so sehr durch die Stahl- und Aluminiumzölle selbst bedroht als durch die potentiell verheerenden Effekte, die ein Zusammenbruch des multilateralen Handelssystems nach sich zöge. Wenn die Nennung angeblicher nationaler Sicherheitsbedrohung ausreicht, um sich von den Beschränkungen des internationalen Handelsrechts zu befreien, dann verliert das System seine Glaubwürdigkeit. Jede Regierung könnte aus kurzfristigen opportunistischen Erwägungen neue Zölle erheben.

Deutschland ist im Vergleich mit anderen großen Volkswirtschaften sehr offen und von möglichst barrierefreiem Zugang zu den internationalen Absatz- und Beschaffungsmärkten abhängig. Nur etwa ein Fünftel der deutschen Exporte außerhalb der EU geht in die Vereinigten Staaten; vier Fünftel gehen an andere WTO-Mitglieder. Daher hat gerade Deutschland ein besonderes Interesse an der Bewahrung eines verlässlichen regelbasierten multilateralen Systems. Die Vergeltungszölle der EU und der anderen Ländern ergeben Sinn, weil sie verhindern, dass die Zollpolitik des amerikanischen Präsidenten für die Vereinigten Staaten volkswirtschaftliche Gewinne hervorbringt.

Die Liste der EU lädt in ihrer Detailliertheit zum Schmunzeln ein; sie ist aber durchaus klug angelegt. Sie umfasst zwei Bereiche: Stahlprodukte und Konsumartikel. Für die Produkte im ersten Teil wird die Handelsumlenkung sehr groß sein. Die Europäer kaufen diese sehr wenig differenzierten Güter einfach anderswo zu Weltmarktprei-

21 Eine genaue Beschreibung der betroffenen Produkte und Länder liefern Felbermayr und Sandkamp (2018). Eine Abschätzung der volkswirtschaftlichen Kosten findet sich bei Felbermayr und Steininger 2018. 
sen ein. Der zweite Teil der Liste enthält meist Markenartikel, bei denen die amerikanischen Anbieter Marktmacht haben. Daher wird bei Jack Daniel's, Estee Lauder und Harley-Davidson die Steuerinzidenz für die EU nicht ganz unvorteilhaft sein. Ein Vergleich der verfügbaren Schätzungen von Exportangebots- und Importnachfrageelastizitäten legt diese Vermutung jedenfalls sehr nahe. Und anekdotische Evidenz über das Preissetzungsverhalten amerikanischer Unternehmen unterstützt die These ebenfalls.

Viele Beobachter haben nicht den Eindruck, dass Trump die Wohlfahrt der Vereinigten Staaten zu maximieren sucht. Dem schließe ich mich an. Für die „Globalist elite“, die mit der Bereitstellung von Dienstleistungen im Silicon Valley oder an der Wallstreet hohe Einkommen erwirtschaftet, interessiert Trump sich nicht. Er hat wohl auch nicht die Kaufkraft der Konsumenten im Allgemeinen im Blick, sondern die Löhne einzelner Gruppen von Industriearbeitern (in der Stahlindustrie oder im Bergbau). Mit seiner nationalistischen Politik kann er sich bisher halten, weil er damit das Ende des amerikanischen Traums - wirtschaftlicher Aufstieg aus eigener Kraft für alle - Ausländern in die Schuhe schieben kann, statt eigene sozial- und wirtschaftspolitische Fehler der Vereinigten Staaten zugeben zu müssen.

Aber die Vereinigten Staaten sind immer noch eine Demokratie. Jene Kräfte, die durch die Zölle und Gegenzölle belastet werden, können erheblichen Druck ausüben. So ist es vielleicht nicht zufällig, dass jüngst eine Gesetzesinitiative, dem Präsidenten die rechtlichen Grundlagen für manche Alleingänge in der Zollpolitik wegzunehmen, von dem republikanischen Senator Robert Corker kam, der aus Tennessee stammt, dem Staat des Bourbon-Whiskey. Bourbon steht auf der Liste der EU und der anderen WTOMitglieder. Auch Politiker aus den reichen demokratischen Staaten an der West- und Ostküste, die vom Dienstleistungsexport leben, werden zunehmend aktiv, weil asymmetrische Vergeltung ihrer Wirtschaft Schaden zufügen würde. Die Erfolgsaussichten der europäischen Strategie sind wohl gar nicht so schlecht.

Der vorläufige handelspolitische Waffenstillstand, der auf dem Gipfel zwischen dem EU Kommissionspräsidenten Juncker und dem amerikanischen Präsidenten Trump erreicht wurde, hat sicher auch mit der konsequenten Haltung der EU zu tun. Japan, das bisher eine sehr konziliante Haltung einnimmt, bleibt hingegen weiterhin von Autozöllen bedroht.

Es gibt noch ein weiteres wichtiges Argument für Vergeltungszölle. Der amerikanischen Regierung geht es darum, das Leistungsbilanzdefizit zu senken. Es ist sehr wahrscheinlich, dass das Defizit 2018 und 2019 steigen wird, weil die Zölle das Grundproblem - eine zu geringe gesamtwirtschaftliche Ersparnis in den Vereinigten Staaten nicht verringern, die nun immer stärker wirkende Steuerreform es aber verstärkt. Es steht zu befürchten, dass der amerikanische Präsident darauf mit weiteren protektionistischen Maßnahmen reagieren könnte. Ein Verzicht auf Vergeltungsmaßnahmen würde ihn darin nur bestärken.

Fazit: Zur Wahrung der deutschen und europäischen Interessen gehören erstens eine konsequente Verteidigung der WTO-Regeln (dazu gehören auch Vergeltungszölle), zweitens die glaubhafte Drohung, bei einer Verschärfung der amerikanischen Politik ebenfalls nachzulegen, und drittens ein ehrliches und praktikables Angebot, mit den Vereinigten Staaten über ein umfassendes bilaterales Handelsabkommen und über die dringend notwendige Reform der WTO zu verhandeln.

Danksagung: Ich danke insbesondere Martin Braml, Clemens Fuest, Hans Gersbach, Martin Hellwig, Karl-Heinz Paqué, Albrecht Ritschl und Jens Südekum für fruchtbaren Gedankenaustausch zu diesem Thema, und Karen Horn für das hervorragende Redigieren der Arbeit.

\section{Literaturverzeichnis}

Bagwell, K. und R. Staiger (1999), An economic theory of GATT, American Economic Review 89(1), S. 215-48.

Bagwell, K. und R. Staiger (2002), The Economics of the World Trading System, Cambridge (MA), MIT Press.

Bagwell, K. und R. Staiger (2016), The design of trade agreements, in: K. Bagwell und R. Staiger (Hrsg.), Handbook of Commercial Policy, Band 1A, Amsterdam, Elsevier, S. 435-529.

Bagwell, K. und R. Staiger (2012), Profit shifting and trade agreements in imperfectly competitive markets, International Economic Review 53(4), S. 1064-104.

Baldwin, R. (2016), The Great Convergence, Cambridge (MA), Harvard University Press.

Bickerdike, C. (1907), Review of A. C. Pigou's Protective and Preferential Import Duties, Economic Journal 17, S. 98-108.

Braml, M. und G. Felbermayr (2018), On the EU-US current account, EconPol Policy Report7.

Broda, C., N. Limão und D. Weinstein (2008), Optimal tariffs: The evidence, American Economic Review 98, S. 2032-65.

Costinot, A. und I. Werning (2018), Lerner symmetry: A modern treatment, American Economic Review: Insights, im Erscheinen.

Felbermayr, G. (2018), Zölle im transatlantischen Handel: Worauf, wieviel, und wie gerecht? Ifo Schnelldienst 71(6), S. 3-8.

Felbermayr, G. und B. Jung (2012), Unilateral trade liberalization in the Melitz model: A note, Economics Bulletin 32(2), S. 1724-30.

Felbermayr, G., B. Jung und M. Larch (2013), Optimal tariffs, retaliation, and the welfare loss from tariff wars in the Melitz model, Journal of International Economics 89(1), S. 13-25.

Felbermayr, G. und A. Sandkamp (2018), Trumps Importzölle auf Stahl und Aluminium, ifo Schnelldienst 71(06), S. 30-37. 
Felbermayr, G. und M. Steiniger (2018), Was kostet der neue USProtektionismus Österreich und der EU? Wirtschaftspolitisches Zentrum Wien, Politikanalyse Nr. 17.

Gros, D. (1987), A note on the optimal tariff, retaliation and the welfare loss from tariff wars in a framework with intra-industry trade, Journal of International Economics 23, S. 357-67.

Grossman, G. und E. Helpman (1994), Protection for sale, American Economic Review 84, S. 833-50.

Holler, M. und G. Illing (2005), Einführung in die Spieltheorie, 6., überarbeitete Auflage, Berlin und Heidelberg, Springer Verlag.

Johnson, H. (1953), Optimum tariff and retaliation, Review of Economic Studies 21, S. 142-53.

Kennan, J. und R. Riezman (1988), Do big countries win tariff wars? International Economic Review 29, S. 81-85.

Krugman, P. (1980), Scale economies, product differentiation, and the pattern of trade, American Economic Review 70, S. 950-59.

Lerner, A. P. (1936), The symmetry between import and export taxes, Economica 3(11), S. 306-13.
Melitz, M. (2003), The impact of trade on intra-industry reallocations and aggregate industry productivity, Econometrica 71, S. 1695725.

Metzler, L. A. (1949), Tariffs, the terms of trade, and the distribution of national income, Journal of Political Economy 57(1), S. 1-29.

Mill, J.S. (1844), Essays on Some Unsettled Questions of Political Economy, London, Parker.

Olson, Mancur (1965), The Logic of Collective Action, Cambridge (MA), Harvard University Press.

Ossa, R. (2011), A 'new trade' theory of GATT/WTO negotiations, Journal of Political Economy 119, S. 122-52.

Ossa, R. (2018), Wie teuer wäre ein Handelskrieg?, Wirtschaftsdienst 98(1), S. 13-15.

Syropoulos, C. (2002), Optimum tariffs and retaliation revisited: How country size matters, Review of Economic Studies 69, S. 707-27.

Venables, A. (1987), Trade and trade policy with differentiated products: A Chamberlainian-Ricardian model, Economic Journal 97, S. 700-17. 